\title{
Splice-Site Mutation
}

National Cancer Institute ( $\mathrm{NCl})$

\section{Source}

National Cancer Institute (NCI). Splice-Site Mutation.

A genetic alteration in the DNA sequence that occurs at the boundary of an exon and an intron (splice site). This change can disrupt RNA splicing resulting in the loss of exons or the inclusion of introns and an altered protein-coding sequence. Also called splice-site variant. 